\title{
Is Assessment of Hyposalivation Important in Routine Dental Practice?
}

\author{
${ }^{1}$ Vasudha Sharma, ${ }^{2}$ Shweta Y Somasundara, ${ }^{3}$ YS Prasanna Kumar, ${ }^{4}$ Padma K Bhat
}

\begin{abstract}
Saliva is a key element in oral homeostasis, oral function and maintenance of oral health. Dry mouth has multiple oral health consequences and affects quality of life. The incidence of dry mouth and its public health impact are increasing due to the aging population, the effects of some systemic diseases, and medical care and commonly prescribed medications that reduce saliva production. Chronic xerostomia can contribute to oral mucosal changes, increased coronal and root caries susceptibility, candidiasis, periodontal disease, partial loss of taste acuity, and difficulty in swallowing and functional prosthetic problems. Although, oral healthcare providers routinely obtain and document a patient's medical history, evaluating salivary gland function is not a common practice unless the patient is symptomatic. This review of literature highlights the importance of saliva, and the need to measure and document hyposalivation in routine dental practice.
\end{abstract}

Keywords: Hyposalivation, Saliva, Xerostomia.

How to cite this article: Sharma V, Somasundara SY, Kumar YSP, Bhat PK. Is Assessment of Hyposalivation Important in Routine Dental Practice? J Health Sci Res 2014;5(2):10-14.

\section{Source of support: Nil \\ Conflict of interest: None}

\section{INTRODUCTION}

Saliva is a key element in oral homeostasis, oral function and maintenance of oral health. Dry mouth has multiple oral health consequences and affects quality of life. The incidence of dry mouth and its public health impact are increasing due to the aging population, the effects of some systemic diseases, and medical care and commonly prescribed medications that reduce saliva production. Adequate salivary secretion is a prerequisite for maintenance of integrity of oral health. Many functions have been ascribed to saliva, including its role as a lubricant that coats and protects the oral tissues against mechanical, thermal and chemical irritants. The high buffering

\footnotetext{
${ }^{1-3}$ Reader, ${ }^{4}$ Professor and Head

${ }^{1-4}$ Department of Public Health Dentistry, Rajarajeswari Dental College and Hospital, Bengaluru, Karnataka, India
}

Corresponding Author: Vasudha Sharma, Reader Department of Public Health Dentistry, Rajarajeswari Dental College and Hospital, Bengaluru, Karnataka, India e-mail:vashu77@gmail.com capacity of saliva tends to keep the oral $\mathrm{pH}$ constant while specific proteins and immunoglobulins offer protection against pathogens. Saliva also provides an appropriate maintenance of teeth, salivary calcium and phosphates help in remineralizing decayed teeth and salivary flow facilitates oral hygiene. All functions are largely protective of oral environment.

Perhaps the best testimony for importance of saliva in oral health is the destruction of oral tissues that occur when saliva and salivary flow are compromised by physiological and pathological states or through medications and treatment. ${ }^{1}$

It is important to the dentist to be knowledgeable about medications and pathological conditions that increase the risk of xerostomia and need for intensive preventive dentistry program. However, the contribution of saliva toward maintenance of oral health is often taken for granted by other healthcare providers, therefore, the dentist is often faced with irreversible consequences of diminished amount of salivary flow and are forced to intervene via extensive dental treatment despite administering excellent dental care.

Although, oral healthcare providers routinely obtain and document a patient's medical history, evaluating salivary gland function is not a common practice unless the patient is symptomatic. ${ }^{2}$ As patient management shifts from surgical to preventive models, the detection, recognition and prevention of salivary gland hypofunction using risk assessment becomes of utmost importance. This review of literature aims to highlight the importance of saliva, and the need to measure and document hyposalivation in routine dental practice.

\section{VARIATIONS IN SALIVARY FLOW RATES}

A healthy person's mean daily saliva production ranges from 1 to 1.51 . The salivary flow index is a parameter allowing stimulated and unstimulated saliva flow to be classified as normal, low or very low (hyposalivation). In adults, normal total stimulated salivary flow ranges from 1 to $3 \mathrm{ml} / \mathrm{min}$, low ranges from 0.7 to $1.0 \mathrm{ml} / \mathrm{min}$, while hyposalivation is characterized by a salivary flow of less than $0.7 \mathrm{ml} / \mathrm{min}$. The normal unstimulated salivary flow ranges from 0.25 to $0.35 \mathrm{ml} / \mathrm{min}$, low ranges from 0.1 to $0.25 \mathrm{ml} / \mathrm{min}$, while hyposalivation is characterized by 
a salivary flow of less than $0.1 \mathrm{ml} / \mathrm{min}$. However, the values denominated 'normal' for stimulated and unstimulated salivary flow exhibit a large biological variation. Thus, individual salivary flow must be monitored regularly and not determined as 'normal' or 'abnormal', based only on one measurement. ${ }^{2}$

The presence of saliva usually is taken for granted, and it is not required for any life sustaining functions. Nevertheless, its diminution or absence can cause significant morbidity and a reduction in a patient's perceptions of quality of life. ${ }^{3}$

A number of physiological circumstances reduce salivary secretion. They include age, the number of teeth in the mouth, male/female, body weight and the time of day. In relation to age, although submaxillary and sublingual gland secretion may be slightly diminished in older persons, the same cannot be said of the parotid glands. Both the parasympathetic and sympathetic nervous systems innervate the salivary glands. Parasympathetic stimulation induces more watery secretions, whereas the sympathetic system produces a sparser and more viscous flow. Therefore, a sensation of dryness may occur, e.g. during episodes of acute anxiety or stress, which cause changes in salivary composition owing to predominant sympathetic stimulation during such periods. Symptoms of a lack of saliva or oral dryness may be precipitated by dehydration of the oral mucosa, which occurs when output by the major and/or minor salivary glands decrease and the layer of saliva that covers the oral mucosa is reduced.

Xerostomia is defined as a subjective complaint of dry mouth that may result from a decrease in the production of saliva. Xerostomia is not a disease but rather a symptom resulting from hyposalivation. Xerostomia was first described by Bartley in 1868. Synonyms of xerostomia include: oligosalia, asialia and stomatitis sicca. ${ }^{4}$

\section{MAIN CAUSES OF HYPOSALIVATION}

Head and neck radiotherapy causes irreversible hyposalivation by destroying the glandular parenchyma. The adverse effects start from 4000 rads onward and the reduction in salivary flow depends on the dose. Doses of less than 10 Gy cause only transient reduction in salivary flow. Doses greater than 10 Gy cause hyposalivation and 15 to 40 Gy cause severe but a reversible reduction in salivary flow. Doses greater than 40 Gy cause irreversible damage to glandular parenchyma followed by atrophy and fibrosis.

Surgical removal of salivary glands because of neoplasms, congenital absence of glands or malformation.

Some systemic disorders cause progressive destruction of the salivary glands, as in some autoimmune diseases, such as Sjögren's syndrome, while others lead to vascular or neurological alterations that have transitory and reversible repercussions on saliva production, as in hypertension, depression, malnutrition, dehydration, diabetes, etc. ${ }^{5}$

Acute viral infections may temporarily decrease salivary flow, anxiety, mental stress may temporarily decrease salivary flow. Hormonal changes, such as those that occur during pregnancy or menopause, could be another cause. Snoring and breathing open mouthed can also be factors. ${ }^{6}$

Medications: ${ }^{7}$ Xerostomia is a common and significant side effect of many commonly prescribed drugs. The risk for xerostomia increases with the number of drugs being taken. Older people, therefore, are more likely to be affected. In the geriatric population, drug-induced xerostomia has been reported to contribute to difficulty with chewing and swallowing; over 400 medicines, many of them in common use, induce salivary gland hypofunction. Some common medications which can cause hyposalivation and xerostomia are as following:

\begin{tabular}{ll}
\hline Medicine group & Examples \\
\hline Anorectic & Fenfluramine \\
Anxiolytics & Lorazepam, diazepam \\
Anticonvulsants & Gabapentin \\
Antidepressants & Tricyclic amitriptyline, imipramine \\
Antidepressants & SSRI sertraline, fluoxetine \\
Antiemetics & Meclizine \\
Antihistaminics & Loratadine \\
Antiparkinsonian & Biperidene, selegiline \\
Antipsychotics & Clozapine, chlorpromazine \\
Bronchodilators & Ipratropium, albuterol \\
Decongestants & Pseudoephedrine \\
Diuretics & Spironolactone, furosemide \\
Muscle relaxants & Baclofen \\
Narcotic analgesics & Meperidine, morphine \\
Sedatives & Flurazepam \\
Antihypertensive & Prazosin hydrochloride \\
Antiarthritic & Piroxicam \\
\hline
\end{tabular}

\section{CLINICAL FEATURES ASSOCIATED WITH HYPOSALIVATION AND XEROSTOMIA}

Clinically, a patient will have dry lips and irritation at the corners of the mouth. The buccal mucosa will be dry, often sticking to a mirror or retraction device. The tongue will be dry and cracked and have a little papillation. It is common to see plaque accumulation, cervical caries, and many previously restored teeth. ${ }^{8}$

Common symptoms of xerostomia may include the following:

- A sticky or burning feeling in the mouth or throat ${ }^{3}$

- Hoarseness ${ }^{6}$

- Cracked lips ${ }^{8}$ 
- Rough tongue ${ }^{8}$

- Trouble chewing, swallowing, tasting, or speaking ${ }^{8}$

- Mouth sores ${ }^{8}$

- Infection in the mouth ${ }^{9}$

Thrush or candidiasis may occur when saliva decreases. Patients who have thrush may have white, cottage cheese-like plaque and reddened tongue..$^{10}$ Patients wearing dentures who have xerostomia may find their prosthetics uncomfortable because the thin film of saliva is not there to help the dentures stick. Xerostomia in these patients can lead to painful denture sores, dry and cracked lips, and increased risks for oral infection. ${ }^{5}$ Also, xerostomia can contribute to halitosis.

\section{SIALOMETRIC EVALUATIONS}

Although, sialometry usually is performed in academic settings, it can be a useful tool for private practitioners to identify patients with salivary gland disorders. As dental research supports further investigations of saliva relationships, the need to measure salivary flow will continue for use as a clinical diagnostic tool and as an epidemiologic research measure. In recent years, salivabased diagnostic tests have been increasing in popularity because of their non-invasive nature.

Salivary flow rates are assessed differently for different purposes. Clinicians commonly use a patient's response to a health questionnaire and the outcome of clinical evaluation as the basis for identification and assessment of dry mouth. Objective measurements of qualitative or quantitative changes in saliva are best captured by collecting saliva from individual glands or from all that contribute to whole saliva. Commonly used stimulants include: gum base, paraffin wax, rubber bands and citric acid.

Several methods for collecting saliva have been reported and tested for validity and reproducibility. Navazesh et $\mathrm{al}^{4}$ compared four traditional assessment methods (suction, swab, spit and draining):

- The suction method used a vacuum suction device with an attached saliva ejector, which was placed under the subject's tongue to extract whole saliva from the mouth into a pre-weighed container.

- The swab method used pre-weighed cotton swabs placed in the floor of the mouth to collect saliva.

- The spit method required the subject to pool saliva in the mouth and then expectorate into a pre-weighed container at specific intervals.

- The accepted 'gold standard' at that time, 'draining' saliva between parted lips into a cup for 10 minutes, was recognized as cumbersome, procedurally distasteful, and prohibitively time consuming for use in large epidemiologic studies.
The study found the swab method to be unreliable and that the suction method had the highest test/retest reliability, but consistently yielded more saliva volume than the spit or draining methods.

The draining method was found comparable to the spit method, and the spit method was preferred by both examiner and subjects.

The authors thus, concluded that the spit and draining methods were preferred over the others for whole saliva flow rate assessment because of their reliability and simplicity, and that the spit method was preferable to the draining method.

Unstimulated saliva: Collecting whole saliva is easier and more cost-effective than collecting saliva from an individual gland (parotids, submandibular/sublingual) in a private practice setup. Whole saliva can be collected under unstimulated (resting) and stimulated conditions.

Patients are instructed not to drink, eat, smoke, perform oral hygiene or put anything into their mouths for 90 minutes before the collection time. The dentist or designated staff member collects the saliva in a quiet environment, with the patient sitting in an upright position, head tilted forward and eyes open, with minimal body and orofacial movements.

The patient is asked to swallow saliva first, then stay motionless and allow the saliva to drain passively for 5 minutes over the lower lip into a test-tube fitted with a funnel. After the 5-minute collection period, the oral healthcare provider asks the patient to void the mouth of saliva by spitting into the funnel.

Stimulated saliva: The clinician then collects stimulated saliva by asking the patient to chew on a piece of gum at approximately 45 chews per minute.

The patient will void the mouth of saliva by spitting into the collection tube every minute for a total of 5 minutes. The clinician, then calculates the salivary flow rate by dividing the amount (weight or volume) of collected saliva by the duration of the collection period (5 minutes).

There is no general agreement about what constitutes a normal salivary flow rate; however, researchers generally consider an unstimulated flow rate of 0.1 to $0.2 \mathrm{ml} / \mathrm{min}$ (or grams per minute) and a chewing stimulated flow rate of $0.7 \mathrm{ml} / \mathrm{min}$ (or $\mathrm{g} / \mathrm{min}$ ) to be abnormally low flow rates. Currently, clinicians use a $0.1 \mathrm{ml} / \mathrm{min}$ unstimulated whole-saliva flow rate as a criterion for the diagnosis of Sjögren's syndrome.

Minor salivary gland biopsy: Histopathologic changes involving the major or minor salivary glands may indicate local or systemic conditions that affect salivary gland secretion. Clinicians can perform a minor salivary gland biopsy in the dental office (using local anesthetic) 
based on the patient's medical status and the clinician's expertise. The common site for a minor salivary gland biopsy is the inner aspect of the lower lip. The histologic changes caused by local or systemic conditions may be reversible or irreversible.

\section{MANAGEMENT OF HYPOSALIVATION}

The management of dry mouth should begin with identification and management of the underlying cause, although this is not always possible. Treatment is directed toward local and systemic salivary gland stimulation, symptomatic relief and preventing and treating complications due to hyposalivation.

- Patients with xerostomia should undergo frequent dental evaluations for early diagnosis of oral complications like dental caries and periodontitis.

- Patients should be encouraged to carry out daily oral self-examinations for any mucosal ulcers, lesions or tooth decay and to report any unusual findings.

- The mainstay of prevention of dental caries is meticulous plaque control through excellent oral hygiene.

- Patients should be instructed to brush their teeth at least twice a day using a soft-bristled toothbrush and a low-abrasive highly fluorinated toothpaste or gel. In addition, use of sodium fluoride rinses or fluoride gel may reduce tooth decay.

- The most suitable topical fluoride gels contain 0.4 to $1.25 \%$ fluoride, have neutral $\mathrm{pH}$ and are applied in mouth guards (custom-made fluoride carriers).

- Patients should be advised against using alcohol and tobacco and to adopt a low-sugar diet to control dental caries. Xylitol-sweetened gums are effective in caries prevention and can be easily incorporated into a patient's routine.

- Salivary stimulation is the preferred treatment in patients with residual capacity in the salivary glands.

- The combination of chewing and taste, as provided by gums and mints, can be effective in relieving symptoms. Citric acid can stimulate salivation, but its use is limited because of attendant mucosal irritation and the risk of demineralization in patients with teeth. A disadvantage of local salivary stimulants is their limited effectiveness during the night when the symptoms are most severe.

Salivary flow rate, buffer effect and perhaps also the in vivo concentrations of some salivary constituents, such as fluoride, hypothiocyanite and agglutinins (possibly including $\operatorname{Ig} \mathrm{A}$ ) seem to be somewhat more important than the others in assessing caries susceptibility and/ or activity. However, the accuracy of any of these parameters alone is poor and, therefore, future research should focus on exploring combinations, or clusters, of salivary parameters which are typical for caries-active and -inactive individuals and populations.

If saliva secretion cannot be stimulated, symptomatic treatment involves the use of saliva substitutes.

- Patients should be encouraged to take frequent sips of water throughout the day. Use of water during meals can aid in swallowing and improve taste perception.

- Commercially available saliva substitutes containing thickening agents, such as carboxymethyl cellulose or mucin, are the most common.

- Recently, saliva substitutes based on polyacrylic acid and xanthan gum have been developed and are recommended for patients with extremely low salivary production rates.

- Although, there is clearly a role for saliva replacements, particularly in those who have no residual salivary gland function, this is not a highly effective therapy. Use of bedside humidifiers, particularly at night, may lessen discomfort due to oral dryness.

- Because of an increase in the survival rate for patients with head and neck cancers and increase in the elderly population, there is an increased need for further research on the alleviation of xerostomia and for the prevention and treatment of the deleterious effects of this condition. ${ }^{7}$

\section{SUMMARY AND CONCLUSION}

Chronic xerostomia can contribute to oral mucosal changes, increased coronal and root caries susceptibility, candidiasis, periodontal disease, partial loss of taste acuity, and difficulty in swallowing and functional prosthetic problems. When reviewing a patient's medical history, dentists should keep in mind these facts: ${ }^{10}$

- Salivary secretion is affected by the nature, severity, number and duration of a variety of medical disorders and medications.

- Women are more susceptible than men to certain medical conditions known to affect salivary secretion.

- Nutritional and dietary habits, as well as oral hygiene practices, have a profound impact on the pattern and severity of oral complications of salivary gland hypofunction.

- Tobacco, alcohol and recreational drugs may affect the quality or quantity of saliva.

- Taking a thorough medical and dental history with regularly recorded updates of medications prescribed is extremely important. It is important for dentists to be knowledgeable about the medications that increase the risk of xerostomia, and the need for an intensive preventive dentistry program. 
- The preventive care program should include patient education in maintaining personal oral hygiene practices, increased fluid intake, professional and self applied fluoride preparations, use of therapeutic agents that stimulate salivation, use of antimicrobial rinses and regularly scheduled dental examinations.

- Pharmacologic agents stimulate salivary output and produce a lasting effect throughout the day. Pilocarpine and cevimeline have been approved for use in xerostomic patients.

\section{REFERENCES}

1. DePaola DP. Saliva: the precious body fluid. J Am Dent Assoc 2008;139(Suppl):5S-6S.

2. Navazesh M. How can oral healthcare providers determine if patients have dry mouth? J Am Dent Assoc 200;134(5):613-620.

3. Spielman A, Ben-Aryeh H, Gutman D, Szargel R, Deutsch E. Xerostomia-diagnosis and treatment. Oral Surg Oral Med Oral Pathol 1981;51(2):144-147.
4. Navazesh M, Christensen CM. A comparison of whole mouth resting and stimulated salivary measurement procedures. J Dent Res 1982;61(10):458-462.

5. Edgar WM. Saliva: its secretion, composition and functions. Br Dent J 1992;172(8):305-312.

6. US Food and Drug Administration Website. For consumers. Dry mouth? Do not delay treatment. Available at: www.fda. gov/ForConsumers/ConsumerUpdates/ucm254273.htm. Accessed March 9, 2013.

7. Llena-Puy C. The rôle of saliva in maintaining oral health and as an aid to diagnosis. Med Oral Patol Oral Cir Bucal 2006;4(5):E449-455.

8. Ciarrocca K, Hupp WS, Firriolo FJ, De Rossi SS. Dry mouth: diagnosis and management. Inside Dental Assisting Website. Available at: http://www.cdeworld.com/courses/4540. Accessed March 9, 2014.

9. Patient guide. Dry mouth. Aegis Communications Website. [Internet] http://www.dentalaegis.com/resources/Patient Guide_Dry_Mouth.pdf. March 9, 2014.

10. Malcmacher L. Xerostomia and the critical role of saliva. Inside Dentistry Web site. [Internet] http://www.dentalaegis. $\mathrm{com} / \mathrm{id} / 2007 / 03 /$ focus-on-xerostomia-and-the-critical-roleof-saliva. Accessed on March 9, 2014. 